Research, Society and Development, v. 9, n. 10, e4149108709, 2020

(CC BY 4.0) | ISSN 2525-3409 | DOI: http://dx.doi.org/10.33448/rsd-v9i10.8709

\title{
Dupla demandas de cuidado do paciente em processo de envelhecimento na internação psiquiátrica
}

Double demands on the care of the aging patient during psychiatric hospitalization

Dobles demandas en el cuidado del paciente anciano durante la hospitalización psiquiátrica

Recebido: 23/09/2020 | Revisado: 29/09/2020 |Aceito: 30/09/2020 | Publicado: 02/10/2020

\section{Emiliane Cunha Ferreira}

ORCID: https://orcid.org/0000-0003-2344-0132

Universidade Federal do Rio de Janeiro, Brasil

E-mail: emilianeipub@gmail.com

Jaqueline Da Silva

ORCID: https://orcid.org/0000-0003-2888-5720

Universidade Federal do Rio de Janeiro, Brasil

E-mail: jaquelinedasilva@ufrj.br

Vivian dos Santos Teixeira

ORCID: https://orcid.org/0000-0003-4236-6716

Universidade Federal do Rio de Janeiro, Brasil

E-mail: vivit.enf@gmail.com

Claudia Barbastefano Monteiro

ORCID: https://orcid.org/0000-0002-3150-7424

Universidade Federal do Rio de Janeiro, Brasil

E-mail: claudia.ipub@gmail.com

\section{Resumo}

O paciente com transtorno mental pode apresentar um processo de envelhecimento acelerado quando comparado com a população em geral. A internação psiquiátrica é um momento de desestruturação da função mental, que pode contribuir para o agravamento de condições clínicas pré-existentes, ou até mesmo o surgimento de comorbidades, aumentando a complexidade do quadro do paciente. Este estudo teve como objetivo investigar as demandas de cuidado em saúde do paciente com transtorno mental em processo de envelhecimento. 
Abordagem quantitativa, com corte temporal e utilização de estatística descritiva simples e medidas de tendência central na análise dos dados. Foi possível comprovar, através dos prontuários coletados, que quase metade dos pacientes apresentava diagnóstico clínico e /ou problemas de saúde relacionados a agravos físicos. Considerando a complexidade do cuidado ao paciente com transtorno mental, que pode apresentar comorbidades de ordem física e está em processo de envelhecimento, é importante a identificação precoce de agravos à saúde física, assim como, a prevenção de doenças crônicas não transmissíveis, durante e após a internação.

Palavras-chave: Hospital psiquiátrico; Envelhecimento; Diagnóstico clínico; Comorbidade.

\begin{abstract}
The patient with mental disorder may experience an accelerated aging process when compared to the general population. Psychiatric hospitalization is a moment of disruption of mental function, which can contribute to the worsening of pre-existing clinical conditions, or even the emergence of comorbidities, increasing the complexity of the patient's condition. This study aimed to investigate the health care demands of aging patients with mental disorders. Quantitative approach, with temporal cut and use of simple descriptive statistics and measures of central tendency in data analysis. It was possible to prove, through the collected medical records, that almost half of the patients had clinical diagnosis and/or health problems related to physical problems. Considering the complexity of care towards aging patients with mental disorders, which can present comorbidities of physical nature, it is important to identify physical health problems early, as well as to prevent chronic noncommunicable diseases, during and after hospitalization.
\end{abstract}

Keywords: Hospitals psychiatric; Aging; Clinical diagnosis; Comorbidity.

\title{
Resumen
}

El paciente con trastorno mental puede experimentar un proceso de envejecimiento acelerado en comparación con la población general. La hospitalización psiquiátrica es un momento de alteración de la función mental, que puede contribuir al empeoramiento de condiciones clínicas preexistentes, o incluso al aparecimiento de comorbilidades, aumentando la complejidad de la condición del paciente. Este estudio tuvo como objetivo investigar las demandas asistenciales de los pacientes con trastornos mentales en el proceso de envejecimiento. Enfoque cuantitativo, con corte temporal y uso de estadística descriptiva simple y medidas de tendencia central en el análisis de datos. Se pudo comprobar, a través de 
la historia clínica recopilada, que casi la mitad de los pacientes tenían diagnóstico clínico y/o problemas de salud relacionados con problemas físicos. Considerando la complejidad de la atención al paciente con trastornos mentales, que pueden presentar comorbilidades de carácter físico y se encuentra en proceso de envejecimiento, es importante identificar precozmente problemas de salud física, así como prevenir enfermedades crónicas no transmisibles, durante y después de la hospitalización.

Palabras clave: Hospital psiquiátrico; Envejecimiento; Diagnóstico clínico; Comorbilidad.

\section{Introdução}

Estudos sobre a história do cuidado em psiquiatria apontam que no passado condições precárias nas unidades de internações e os tratamentos dispensados aos pacientes psiquiátricos eram causas importantes pela excessiva mortalidade precoce dos internos (Henna, 2015).

Com o passar dos anos e de avanços nos diagnósticos e tratamentos e reorientação do modelo assistencial no campo da saúde mental houve melhoria de condições de tratamento ao paciente psiquiátrico. Entretanto, resultados continuam a evidenciar a mortalidade precoce de pacientes psiquiátricos que embora menos elevada, ainda é maior quando comparadas com a população em geral (Baeza, 2017).

Pessoas com transtornos mentais possuem um risco 2.2 vezes mais elevado de morrer por qualquer causa e a média de anos de vida perdidos para esta população é de 10 anos (Walker, McGee, \& Druss, 2015).

Mais uma preocupação, é o fato de que não sabemos a dimensão real do problema no que se refere ao paciente com transtorno mental que tem comorbidades de ordem física e viceversa. Essencialmente porque não se tem como cruzar dados com as informações disponíveis na literatura (Baeza, 2017).

À luz da Lei 8.080, em conformidade com os princípios do Sistema Único Saúde (SUS), de universalidade, integralidade e equidade, e em contexto da consolidação da Reforma Psiquiátrica, não há como separar o corpo da mente de pacientes com transtorno mental que passam por eventuais ou frequentes internações (M. da S. Brasil, 1990).

$\mathrm{Na}$ internação psiquiátrica faz -se necessário um olhar atento para os indivíduos, em crise psiquiátrica e com comorbidades clínicas para que seja possível o acolhimento desta clientela, sob a perspectiva da Reforma Psiquiátrica, preconizado pela lei 10216/2001 de igualdade, equidade e direito a reinserção e reabilitação psicossocial (P. da R. Brasil, 2001). 
Este estudo foi motivado a partir da observação no cotidiano da prática assistencial em uma unidade de internação psiquiátrica no ano de 2014, e parte do pressuposto que existe dupla demandas de cuidado do paciente com transtorno mental em processo de envelhecimento: a demanda psíquica e a física.

O paciente com transtorno mental pode apresentar um processo de envelhecimento acelerado quando comparado com a população em geral. O transtorno bipolar apresenta fatores que contribuem para um envelhecimento diferenciado quando comparado com pessoas que não apresentam distúrbios psiquiátricos (Panizzutti, 2016).

O uso prolongado de múltiplas medicações, mais de um agravo à saúde, as condições precárias de tratamento, e o estresse são fatores que predispõe um envelhecimento acelerado para estes pacientes.

$\mathrm{Na}$ admissão no hospital psiquiátrico, alguns pacientes podem chegar sedados, agitados ou tão desorganizados que nem mesmo têm condições de informar sobre si ou contatos de familiares. Em tais situações, podem ocorrer que dados de ordem pessoal e de saúde iniciais sejam coletados tardiamente junto a visitantes e / ou terceiros como amigos, vizinhos.

Ainda do ponto de vista clínico, outro desafio real a ser considerado para a realização do estudo em tela, é que os pacientes com transtornos mentais graves podem ter dificuldade em comunicar objetivamente suas queixas somáticas. Podem não ser adequadamente “ouvidos" pelos médicos em geral, pois o estigma de "louco" invalida suas queixas somáticas (Dalgalarrondo, 2019).

Um estudo apontou que os profissionais de saúde apresentaram tendência em focar na intervenção da comorbidade ou do transtorno mental. Mostrou também falha de comunicação com pacientes e familiares, o não monitoramento da comorbidade bem como a ausência de conhecimento sobre ela. Apontou equipes desprovidas para o atendimento as queixas físicas que foram, por vezes, classificadas como sintomas do transtorno mental (Assis, 2019).

Não menos importante, é o fato de que, estes pacientes ainda têm dificuldades em acessar com dignidade alguns serviços de saúde, pelo estigma que a doença mental produz, principalmente entre profissionais de saúde, o que dificulta o diagnóstico precoce de condições clínicas, como hipertensão, diabetes mellitus e neoplasias (Nascimento \& Leão, 2019).

Pacientes com transtornos mentais e doenças crônicas concomitantes estão em maior risco e são mais propensos a precisar de cuidados intensivos do que os pacientes com doenças crônicas isoladamente (Abernathy et al., 2016) 
Estudos comprovam que pacientes psiquiátricos apresentam mortalidade precoce quando comparados com a população em geral, dentre as causas as doenças cardiovasculares se destacam. Fatores associados ao estilo de vida e a efeitos adversos dos medicamentos utilizados pelos pacientes podem estar associados à maior mortalidade (Baeza, 2017).

Considerando que a internação psiquiátrica é um momento de desestruturação da função mental, que pode contribuir para o agravamento de condições clínicas pré-existentes, ou até mesmo o surgimento de comorbidades, aumentando a complexidade do quadro do paciente, este estudo teve como objetivo investigar as demandas de cuidado em saúde do paciente com transtorno mental em processo de envelhecimento.

\section{Metodologia}

Estudo de abordagem quantitativa, com corte temporal e estatística descritiva simples e medidas de tendência central para tratamento e análise dos dados. Os procedimentos estatísticos permitem que a pesquisadora resuma, organize, interprete e comunique a informação numérica - na forma de subsídios tratados para utilização em favor da equipe e dos pacientes da instituição cenário (Polit \& Beck, 2019). O aspecto matemático pode ser usado em vários momentos, ele torna-se importante por prever os acontecimentos, inclusive na área da saúde. Empregam-se métodos estatísticos para alcançar previsões em relação a algum fenômeno em estudo (Pereira, Shitsuka, Parreira, \& Shitsuka, 2018)

O cenário foi uma instituição psiquiátrica universitária, localizada no município do Rio de Janeiro, com 106 leitos psiquiátricos sendo distribuídos em: 56 leitos femininos e 50 leitos masculinos.

A coleta de dados foi por consulta documental realizada no ano de 2014, em prontuários e impressos de pacientes com transtornos mentais internados na unidade psiquiátrica, no período compreendido entre 2010 a 2012 a fim de realizar o levantamento das demandas de cuidados durante o período de internação.

Em observância aos princípios bioéticos de autonomia, beneficência, não-maleficência e justiça contidos na Resolução 466/12 do Conselho Nacional de Saúde (CNS) do Brasil, foi solicitado autorização institucional para a coleta de dados quantitativos sendo aprovado pelo Comitê de Ética e Pesquisa da Escola de Enfermagem Anna Nery Hospital/Escola São Francisco de Assis (EEAN/HESFA/UFRJ) sob o parecer $n^{\circ}: 377.875$ e Comitê de Ética e Pesquisa do Instituto de Psiquiatria da Universidade Federal do Rio de Janeiro (IPUB/UFRJ) sob o parecer $n^{\text {o: }} 437.773$. 


\section{Resultados e Discussão}

A partir da observação do cotidiano da prática, emergiram inquietações sobre as demandas de cuidados em saúde para o paciente que: interna com um agravo de ordem mental, está em processo de envelhecimento e pode apresentar comorbidades físicas, reafirmando a complexidade da assistência. Através do levantamento de dados dos pacientes com transtornos mentais que internaram no período de 2010 a 2012, podemos fazer as afirmações abaixo.

O número total corresponde aos prontuários localizados, foi de 1354, conforme detalhado no Quadro 1 abaixo.

Quadro 1. Distribuição da População do Estudo.

\begin{tabular}{|l|l|}
\hline Ano & $\begin{array}{l}\text { Prontuários de internação psiquiátrica } \\
\text { localizados }\end{array}$ \\
\hline 2010 & 427 \\
\hline 2011 & 460 \\
\hline 2012 & 467 \\
\hline Total & 1.354 \\
\hline
\end{tabular}

Fonte: A autora, (2014).

Nota -se pelo quadro acima que no ano de 2010 de um total de 427 prontuários de internação localizados $243(56,9 \%)$ ou pouco mais de metade foram de mulheres, quando comparados ao número de homens, correspondendo a $184(43,1 \%)$ dos casos.

Para o ano de 2011 de um total de 460 prontuários de internação localizados 236 (51,3\%) foram de homens, e $224(48,7 \%)$ de mulheres, portanto uma distribuição equilibrada entre os sexos.

Os dados de 2012 revelam que de um total de 467 prontuários de internação localizados $249(53,3, \%)$ foram de homens, e 218 (46,7\%) de mulheres, portanto também uma distribuição equilibrada entre os sexos.

Em 2010, na variável diagnóstico psiquiátrico dentre 427, os agravos à saúde mental mais frequentes foram: o transtorno bipolar do humor responsável por $194(45,5 \%)$ das internações, a esquizofrenia com $140(32,7 \%)$ o transtorno de personalidade $33(7,8 \%)$, outros transtornos psiquiátricos somaram $60(14 \%)$. 
Research, Society and Development, v. 9, n. 10, e4149108709, 2020

(CC BY 4.0) | ISSN 2525-3409 | DOI: http://dx.doi.org/10.33448/rsd-v9i10.8709

Em relação ao ano de 2011, dentre 460, os agravos à saúde mental mais frequentes foram: o transtorno bipolar responsável por 206 (44,8\%) das internações, a esquizofrenia com $153(33,2 \%)$, o transtorno de personalidade 49 (10,7\%), outros transtornos psiquiátricos 52 $(11,3 \%)$.

Em 2012, na variável diagnóstico psiquiátrico dentre 467, os agravos à saúde mental mais frequentes foram: o transtorno bipolar foi responsável por $214(45,8 \%)$ das internações, a esquizofrenia com 142 (30,5\%), o transtorno de personalidade 20 (4,2\%), e outros transtornos psiquiátricos $91(19,5 \%)$.

Em 2010 a variável clínica identificou 224 (57,1\%) agravos à saúde física, as principais foram: as doenças do aparelho circulatório 84 (37,5\%), a cefaleia 74 (33\%). Embora a cefaleia seja considerada um sintoma é classificada pela CID-10 como diagnóstico, por este motivo no presente estudo foi apresentada na variável diagnóstico clínico. O restante $29,5 \%$, foi dividido em doenças do aparelho digestivo, doenças do sistema osteomusculares e tecidos conjuntivos e as doenças infecciosas.

Para o ano de 2011 a variável diagnóstico clínico identificou 220 (47,8\%) de agravos à saúde física, as principais foram: as doenças do aparelho circulatório 89 (40,5\%), a cefaleia 63 $(28,6 \%)$, as doenças endócrinas, nutricionais e metabólicas as doenças do aparelho digestivo e as doenças infecciosas somaram $30,9 \%$.

Em 2012 a variável clínica identificou 258 (55,3\%) agravos à saúde física, as principais foram: a cefaleia $110(42,6 \%)$, as doenças do aparelho circulatório 77 (29,8\%), as doenças endócrinas, nutricionais e metabólicas e as doenças infecciosas somaram 27,5\%.

Para melhor visualização no Quadro 2 observa-se uma síntese do descrito anteriormente. 
Quadro 2. Síntese dos achados nos prontuários.

\begin{tabular}{|l|l|l|l|l|l|l|}
\hline Variável/ Ano & \multicolumn{2}{l}{$\mathbf{2 0 1 0}$} & \multicolumn{2}{l|}{$\mathbf{2 0 1 1}$} & \multicolumn{2}{l|}{$\mathbf{2 0 1 2}$} \\
\hline Sexo & $\mathbf{N}^{\mathbf{0}}$ & $\mathbf{\%}$ & $\mathbf{N}^{\mathbf{0}}$ & $\mathbf{\%}$ & $\mathbf{N}^{\mathbf{o}}$ & $\mathbf{\%}$ \\
\hline Masculino & 184 & 43,1 & 236 & 51,3 & 249 & 53,3 \\
\hline Feminino & 243 & 56,9 & 224 & 48,7 & 218 & 46,9 \\
\hline Total & 427 & 100 & 460 & 100 & 467 & 100 \\
\hline Diagnóstico Psiquiátrico & $\mathbf{N}^{\mathbf{0}}$ & $\mathbf{\%}$ & $\mathbf{N}^{\mathbf{0}}$ & $\mathbf{\%}$ & $\mathbf{N}^{\mathbf{0}}$ & $\%$ \\
\hline Transtorno do humor & 194 & 45,5 & 206 & 44,8 & 214 & 45,8 \\
\hline Esquizofrenia & 140 & 32,7 & 153 & 33,2 & 142 & 30,5 \\
\hline Transtorno de personalidade & 33 & 7,8 & 49 & 10,7 & 20 & 4,2 \\
\hline Outros transtornos psiquiátricos & 60 & 14 & 52 & 11,3 & 91 & 19,5 \\
\hline Total & 427 & 100 & 460 & 100 & 467 & 100 \\
\hline Diagnóstico clínico & $\mathbf{N}^{\mathbf{0}}$ & $\mathbf{\%}$ & $\mathbf{N}^{\mathbf{0}}$ & $\mathbf{\%}$ & $\mathbf{N}^{\mathbf{0}}$ & $\%$ \\
\hline Agravos a saúde física & 244 & 57,1 & 220 & 47,8 & 258 & 55,2 \\
\hline Doenças do aparelho circulatório & 84 & 37,5 & 89 & 40,5 & 77 & 29,8 \\
\hline Cefaleia & 74 & 33 & 63 & 28,6 & 110 & 42,6 \\
\hline Outros & 83 & 29,5 & 68 & 30,9 & 71 & 27,5 \\
\hline Total & 427 & 100 & 460 & 100 & 467 & 100 \\
\hline
\end{tabular}

Fonte: A autora, (2014).

Observa-se no Quadro 2, o elevado número de diagnósticos clínicos evidencia a complexidade do paciente que interna com comorbidades de ordem física além de um ou mais agravos à saúde mental, causa inicial de sua admissão. O Quadro 3 ilustra este achado.

Quadro 3. Comparativo de internações psiquiátricas e agravos físicos.

\begin{tabular}{|l|l|l|}
\hline Ano & $\begin{array}{l}\text { Prontuários de internação } \\
\text { psiquiátrica localizados }\end{array}$ & Agravos físicos \\
\hline 2010 & 427 & 244 \\
\hline 2011 & 460 & 220 \\
\hline 2012 & 467 & 258 \\
\hline Total & 1.354 & 722 \\
\hline
\end{tabular}

Fonte: A autora, (2014). 
Detalha-se que no Quadro 3, que das 1354 internações psiquiátricas nos anos de 2010, 2011 e 2012, mais de 50\% (722) dos pacientes apresentou agravos físicos a saúde, o que significa que além da demanda psíquica existiu a demanda física.

Entre os adultos com diagnóstico de doença mental, 68\% também tinham um ou mais problemas médicos crônicos doenças. Dados mostraram que a coexistência de transtornos mentais e doenças crônicas está associada à baixa adesão ao tratamento, piores resultados e aumento da mortalidade. Além disso, pacientes com transtornos mentais não tratados têm maior probabilidade de desenvolver hipertensão, obesidade ou diabetes, o que aumenta substancialmente a morbidade e os custos com saúde (Abernathy et al., 2016).

A base gerada no estudo configura documentação de dados inéditos, informativos e indicativos da ainda maior complexidade do paciente e de suas demandas. Infelizmente, não passíveis de comparação junto aos dados da base Departamento de Informática do Sistema Único de Saúde (DATASUS) por esta não os ter elencados ou disponíveis para fins de contraste em relação ao Brasil e regiões. No DATASUS, só é possível visualizar o diagnóstico psiquiátrico de forma isolada.

Não há como fazer o cruzamento e comparação dos dados de pacientes que internam por problemas psiquiátricos e também possuem problemas de ordem física. Deve-se ressaltar que além de demandar maior investimento de tempo, de equipe e financeiros em seu atendimento, também estão em processo de envelhecimento com estressores biológicos além dos comportamentais, possuem diagnóstico psiquiátrico e clínico que, se não identificados e tratados a tempo podem levar a óbito.

\section{Considerações Finais}

Este estudo foi motivado a partir da observação no cotidiano da prática assistencial em uma unidade de internação psiquiátrica. As intercorrências físicas apresentadas por pacientes psiquiátricos, durante o período de internação, conduziram a uma reflexão sobre os cuidados que envolviam a assistência. Por se tratar, de um paciente que apresenta potencial para o comprometimento de agravos à sua saúde física, e está em processo de envelhecimento possivelmente acelerado quando comparado com a população em geral.

A observação na prática, versava sobre a possível presença de comorbidade física no paciente com transtorno mental, aumentando a complexidade do cuidado. O que foi possível comprovar com apenas $60 \%$ do total de prontuários coletados, em que quase metade dos 
pacientes apresentava diagnóstico clínico e /ou problemas de saúde relacionados a agravos físicos.

Aponta-se dentre os transtornos psiquiátricos, os mais frequentes foram o transtorno bipolar e a esquizofrenia, sendo a esquizofrenia em 2010-2011, predominante entre homens de meia idade (34 a 49 anos) e o transtorno bipolar em mulheres de mesma faixa etária. Para o ano de 2012 houve predomínio de internações de homens com esquizofrenia e transtorno bipolar, jovens (18 a 33 anos). Em relação ao diagnóstico clínico, as doenças do aparelho circulatório foram mais observadas em mulheres nas faixas etárias de 34 a 49 e 50 a 65 anos, do que em homens das mesmas faixas etárias. As doenças endócrinas, nutricionais e metabólicas no período estudado foram mais frequentes nas mulheres na faixa etária de 50 a 65 anos, do que em homens para a mesma faixa etária.

Concluímos que os pacientes internados na instituição apresentam múltiplas demandas de cuidados tanto de ordem mental quanto física, confirmados pelos diagnósticos psiquiátricos e clínicos. Portanto, considerando a complexidade do cuidado ao paciente com transtorno mental, que pode apresentar comorbidades de ordem física e está em processo de envelhecimento, é importante a identificação precoce de agravos à saúde física, assim como, a prevenção de doenças crônicas não transmissíveis, durante e após a internação. Acreditamos também, ser necessária a construção de propostas terapêuticas concretas que visualizem as múltiplas dimensões do cuidado que envolvem o paciente com transtorno mental.

Atuação assistencial em saúde mental, exige iniciativas em educação permanente, não só sobre cuidados em saúde mental, mas também sobre agravos à saúde física dos pacientes com transtorno mental. Reiterando, portanto, a necessidade de estudar demandas de cuidados em saúde, na vertente da integralidade e baseados em evidências dos pacientes com transtorno mental.

Acredita-se, ser necessária a construção de propostas terapêuticas concretas que visualizem as múltiplas dimensões do cuidado que envolvem o paciente com transtorno mental. Assim como, a implementação de práticas de educação permanente que contribuam com a formação de todos os profissionais envolvidos na assistência. Como proposta futura a criação de um formulário de admissão e alta multidimensional para atender a demandas e direitos ao cuidado integral em saúde, de pacientes portadores de transtorno mental nas diversas fases do ciclo vital, em situações de alta e admissão hospitalar em psiquiatria. 


\section{Referências}

Abernathy, K., Zhang, J., Mauldin, P., Moran, W., Abernathy, M., Brownfield, E., \& Davis, K. (2016). Acute care utilization in patients with concurrent mental health and complex chronic medical conditions. Journal of Primary Care \& Community Health, 7(4), 226-233. https://doi.org/10.1177/2150131916656155

Assis, A. D. De. (2019). Itinerário terapêutico em situação de comorbidade: desafios para a integralidade do cuidado em saúde mental. Cadernos Brasileiros de Saúde Mental, 11(29), 33-46.

Baeza, F. L. C. (2017). Desfechos negativos entre pacientes internados em unidade psiquiátrica de hospital geral: um estudo longitudinal. Universidade Federal do Rio Grande do Sul.

Brasil, M. da S. Lei 8.080 de 19 de setembro de 1990 (1990).

Brasil, P. da R. Lei 10216, de 6 de abril de 2001 (2001).

Dalgalarrondo, P. (2019). Psicopatologia e Semiologia dos Transtornos Mentais. (Artmed, Org.) ( $3^{\text {a }}$. São Paulo.

Henna, E. S. (2015). Experiências narradas: caminhos da loucura entre hospitais psiquiátricos e serviços comunitários de saúde mental (Grande ABC/São Paulo, 1988-2014). Casa de Oswaldo Cruz - FIOCRUZ.

Nascimento, L. A. do, \& Leão, A. (2019). Estigma social e estigma internalizado: a voz das pessoas com transtorno mental e os enfrentamentos necessários. História, Ciências, SaúdeManguinhos, 26(1), 103-121. https://doi.org/10.1590/s0104-59702019000100007

Panizzutti, B. S. (2016). O papel dos marcadores biológicos como indicativos de envelhecimento precoce no Transtorno Bipolar. Universidade Federal do Rio Grande do Sul Porto Alegre. 
Pereira, A. S., Shitsuka, D. M., Parreira, F. J., \& Shitsuka, R. (2018). Metodologia da pesquisa científica. (U. UFSM, NTE, Org.), Metodologia da Pesquisa Científica. Santa Maria

RS.

Recuperado de

https://repositorio.ufsm.br/bitstream/handle/1/15824/Lic_Computacao_Metodologia-

Pesquisa-Cientifica.pdf?sequence=1. Acesso em: 30 setembro 2020.

Polit, D. F., \& Beck, C. T. (2019). Fundamentos de Pesquisa em Enfermagem: Avaliação de Evidências para a Prática da Enfermagem (9ºd). São Paulo: Artmed.

Walker, E. R., McGee, R. E., \& Druss, B. G. (2015). Mortality in Mental Disorders and Global Disease Burden Implications. JAMA Psychiatry, 72(4), 334. https://doi.org/10.1001/jamapsychiatry.2014.2502

\section{Porcentagem de contribuição de cada autor no manuscrito}

Emiliane Cunha Ferreira-40\%

Jaqueline da Silva-20\%

Vivian dos Santos Teixeira $-20 \%$

Claudia Barbastefano Monteiro- 20\% 